\title{
On Karatsuba Conjecture and the Lindelöf Hypothesis
}

\author{
by \\ ShaO-Ji Feng (Beijing)
}

1. Introduction. Let $s=\sigma+i t$ be a complex variable, and $\zeta(s)$ the Riemann zeta function. An interesting problem in the theory of the Riemann zeta function is to obtain lower estimates of the function

$$
F(T ; \Delta ; \sigma)=\max _{T \leq t \leq T+\Delta}|\zeta(\sigma+i t)|
$$

for a fixed $\sigma, 1 / 2 \leq \sigma \leq 1$, where $\Delta=\Delta(T)$ decreases with $T$. Many important results in this area are described in $[2,6]$.

The most interesting case is $\sigma=1 / 2$. Set

$$
F(T ; \Delta)=F(T ; \Delta ; 1 / 2), \quad G(T ; \Delta)=\max _{\left|s-s_{0}\right|=\Delta}|\zeta(s)|,
$$

where $s_{0}=1 / 2+i T$. Karatsuba $[3,4]$ considered the behavior of $F(T ; \Delta)$ and $G(T ; \Delta)$ for $\Delta=\Delta(T) \rightarrow 0$ as $T \rightarrow \infty$. He stated the following conjectures:

ConjeCture 1. There exist a constant $A>0$ and a function $\Delta=$ $\Delta(T) \rightarrow 0$ as $T \rightarrow \infty$, such that for $T$ large enough,

$$
F(T ; \Delta) \geq T^{-A} .
$$

Conjecture 2. Conjecture 1 is valid for $\Delta=(\log \log T)^{-1}$.

Conjecture 3. Conjecture 1 is valid for $\Delta=(\log T)^{-1}$.

CONJeCture $1^{\prime}$. There exist a constant $A>0$ and a function $\Delta=$ $\Delta(T) \rightarrow 0$ as $T \rightarrow \infty$, such that for $T$ large enough,

$$
G(T ; \Delta) \geq T^{-A} \text {. }
$$

Conjecture $2^{\prime}$. Conjecture $1^{\prime}$ is valid for $\Delta=(\log \log T)^{-1}$.

Conjecture $3^{\prime}$. Conjecture $1^{\prime}$ is valid for $\Delta=(\log T)^{-1}$.

2000 Mathematics Subject Classification: Primary 11M06.

Key words and phrases: Riemann zeta function, Karatsuba Conjecture, Lindelöf Hypothesis. 
Obviously for $N=1,2,3$ Conjecture $N$ implies Conjecture $N^{\prime}$, and Conjecture 3 implies all the other conjectures.

In [2], Garaev proved

Theorem A. Conjecture $3^{\prime}$ is equivalent to Conjecture 3.

It is known that the Riemann hypothesis

all the complex zeros of $\zeta(s)$ lie on the critical line $\sigma=1 / 2$

implies Conjecture 3 and thus all the Karatsuba conjectures. This can be proved by using [6, Theorems 14.13 and 14.15] (see [2] for the details).

The Lindelöf Hypothesis is that

$$
\zeta(1 / 2+i t)=O\left(t^{\varepsilon}\right)
$$

as $t \rightarrow \infty$ for every positive $\varepsilon$. There are many equivalent forms of the Lindelöf Hypothesis; one of them is the following.

Theorem B ([6, Theorem 13.5]). A necessary and sufficient condition for the truth of the Lindelöf Hypothesis is that, for every $\sigma>1 / 2$,

$$
N(\sigma, T+1)-N(\sigma, T)=o(\log T),
$$

where $N(\sigma, T)$ is the number of zeros $\beta+i \gamma$ of the Riemann zeta function such that $\beta>\sigma, 0<t \leq T$.

By (1.1), we see that the Lindelöf Hypothesis is equivalent to a much less drastic hypothesis about the distribution of the zeros than the Riemann Hypothesis.

A natural question is: what is the relation between the Karatsuba Conjectures and Lindelöf Hypothesis? In this paper, we will prove

Theorem 1. On the Lindelöf Hypothesis, Conjecture 1 is valid for an arbitrary given constant $A>0$.

The proof of Theorem 1 is based on the following.

Theorem 2. On the Lindelöf Hypothesis, as $T \rightarrow \infty$,

$$
\max _{0 \leq c \leq 1}\left|\int_{T}^{T+c} \log \right| \zeta(1 / 2+i t)|d t|=o(\log T) .
$$

2. Proof of Theorem 2. To prove Theorem 2, we need the following lemmas.

Lemma 1. On the Lindelöf Hypothesis, as $T \rightarrow \infty$,

$$
\arg \zeta(\sigma+i T)=o(\log T)
$$

uniformly for $\sigma \in[1 / 2,2)$, where, if $T$ is not the ordinate of a zero of $\zeta(s)$, the value of $\arg \zeta(\sigma+i T)$ is obtained by continuous variation along the 
straight lines joining $2,2+i T, \sigma+i T$, starting with the value 0 ; if $T$ is the ordinate of a zero of $\zeta(s)$,

$$
\arg \zeta(\sigma+i T)=\lim _{t \rightarrow T+0} \arg \zeta(\sigma+i t) .
$$

Proof. We refer to Cramér [1], who proved the case $\sigma=1 / 2$, and the proof also applies to the case $1 / 2<\sigma<2$.

LEMma 2. On the Lindelöf Hypothesis, as $T \rightarrow \infty$,

$$
\int_{1 / 2}^{1}(N(\sigma, T+1)-N(\sigma, T)) d \sigma=o(\log T) .
$$

Proof. Let

$$
f(\sigma, T)=\frac{N(\sigma, T+1)-N(\sigma, T)}{\log T} .
$$

By Theorem B, we have

$$
\lim _{T \rightarrow \infty} f(\sigma, T)=0, \quad \forall \sigma \in(1 / 2,1] .
$$

By [6, Theorem 9.2], we have

$$
f(\sigma, T) \leq f(1 / 2, T) \leq M, \quad \forall T>0, \sigma \in(1 / 2,1],
$$

where $M$ is an absolute constant. Thus by the Lebesgue Theorem, we get

$$
\lim _{T \rightarrow \infty} \int_{1 / 2}^{1} f(\sigma, T) d \sigma=\int_{1 / 2}^{1} \lim _{T \rightarrow \infty} f(\sigma, T) d \sigma=\int_{1 / 2}^{1} 0 d \sigma=0 .
$$

The proof is complete.

We next need a general formula concerning the zeros of an analytic function in a rectangle, due to Littlewood.

LEMma 3 (see $[5,6])$. Suppose that $\phi(s)$ is meromorphic in and upon the boundary of a rectangle bounded by the lines $t=0, t=T, \sigma=\alpha, \sigma=\beta$ $(\beta>\alpha)$, and regular and not zero on $\sigma=\beta$. The function $\log \phi(s)$ is regular in the neighborhood of $\sigma=\beta$, and here, starting with any value of the logarithm, we define $F(s)=\log \phi(s)$. For other points $s$ of the rectangle, we define $F(s)$ to be the value obtained from $\log \phi(\beta+i t)$ by continuous variation along $t=$ constant from $\beta+$ it to $\alpha+i t$, provided that the path does not cross a zero or pole of $\phi(s)$; if it does, we put

$$
F(s)=\lim _{\varepsilon \rightarrow 0} F(\sigma+i t+i \varepsilon) .
$$

Let $\nu\left(\sigma^{\prime}, T\right)$ denote the excess of the number of zeros of $\phi(s)$ over the number of poles of $\phi(s)$ in the part of the rectangle for which $\sigma>\sigma^{\prime}$, including zeros 
or poles on $t=T$, but not those on $t=0$. Then

$$
\int F(s) d s=-2 \pi i \int_{\alpha}^{\beta} \nu(\sigma, T) d \sigma,
$$

where the first integral is taken around the rectangle in the positive direction.

Proof of Theorem 2. Applying Lemma 3 with $\phi(s)=\zeta(s), \alpha=1 / 2$, $\beta=2$, and taking the imaginary part of (2.1), we get

$$
\begin{aligned}
\int_{0}^{T} \log |\zeta(1 / 2+i t)| d t= & \int_{0}^{T} \log |\zeta(2+i t)| d t \\
& -\int_{1 / 2}^{2} \arg \zeta(\sigma+i T) d \sigma-2 \pi \int_{1 / 2}^{1} N(\sigma, T) d \sigma,
\end{aligned}
$$

where the value of $\arg \zeta(\sigma+i T)$ is defined in Lemma 1. Replacing $T$ with $T+c$ in (2.2), we obtain

$$
\begin{aligned}
\int_{0}^{T+c} \log |\zeta(1 / 2+i t)| d t & =\int_{0}^{T+c} \log |\zeta(2+i t)| d t \\
& -\int_{1 / 2}^{2} \arg \zeta(\sigma+i(T+c)) d \sigma-2 \pi \int_{1 / 2}^{1} N(\sigma, T+c) d \sigma .
\end{aligned}
$$

(2.3) minus (2.2) gives

$$
\begin{array}{r}
\int_{T}^{T+c} \log |\zeta(1 / 2+i t)| d t=-\int_{1 / 2}^{2}(\arg \zeta(\sigma+i(T+c))-\arg \zeta(\sigma+i T)) d \sigma \\
+\int_{T}^{T+c} \log |\zeta(2+i t)| d t-2 \pi \int_{1 / 2}^{1}(N(\sigma, T+c)-N(\sigma, T)) d \sigma
\end{array}
$$

Hence

$$
\begin{aligned}
& \max _{0 \leq c \leq 1}\left|\int_{T}^{T+c} \log \right| \zeta(1 / 2+i t)|d t| \\
& \leq \max _{0 \leq c \leq 1}\left|\int_{1 / 2}^{2}(\arg \zeta(\sigma+i(T+c))-\arg \zeta(\sigma+i T)) d \sigma\right| \\
& \quad+\max _{0 \leq c \leq 1}\left|\int_{T}^{T+c} \log \right| \zeta(2+i t)|d t| \\
& +\max _{0 \leq c \leq 1}\left|2 \pi \int_{1 / 2}^{1}(N(\sigma, T+c)-N(\sigma, T)) d \sigma\right|
\end{aligned}
$$


By Lemma 1,

$$
\begin{aligned}
& \max _{0 \leq c \leq 1}\left|\int_{1 / 2}^{2}(\arg \zeta(\sigma+i(T+c))-\arg \zeta(\sigma+i T)) d \sigma\right| \\
& \quad \leq \int_{1 / 2}^{2}\left(\max _{0 \leq c \leq 1}|\arg \zeta(\sigma+i(T+c))|+|\arg \zeta(\sigma+i T)|\right) d \sigma=o(\log T) .
\end{aligned}
$$

Let $\Lambda(n)=\log p$ if $n$ is $p$ or a power of $p$, and otherwise $\Lambda(n)=0$, and let $\Lambda_{1}(n)=\Lambda(n) / \log n$. We have

$$
\log \zeta(2+i t)=\sum_{n=2}^{\infty} \frac{\Lambda_{1}(n)}{n^{2+i t}} .
$$

Hence

$$
\begin{aligned}
& \max _{0 \leq c \leq 1}\left|\int_{T}^{T+c} \log \right| \zeta(2+i t)|d t| \\
& \quad=\max _{0 \leq c \leq 1}\left|\operatorname{Re} \sum_{n=2}^{\infty} \frac{\Lambda_{1}(n)}{n^{2}} \frac{n^{-i(T+c)}-n^{-i T}}{-i \log n}\right|=O(1)=o(\log T) .
\end{aligned}
$$

By Lemma 2,

$$
\begin{aligned}
\max _{0 \leq c \leq 1}\left|2 \pi \int_{1 / 2}^{1}(N(\sigma, T+c)-N(\sigma, T)) d \sigma\right| \\
=2 \pi \int_{1 / 2}^{1}(N(\sigma, T+1)-N(\sigma, T)) d \sigma=o(\log T) .
\end{aligned}
$$

Combining (2.4)-(2.7), we get (1.2).

3. Proof of Theorem 1. By Theorem 2, there exists a function $h(T)$ such that

$$
\begin{gathered}
h(T)>0, \quad \forall T>1, \\
\lim _{T \rightarrow \infty} h(T)=0
\end{gathered}
$$

and for all $T>1$,

$$
\max _{0 \leq c \leq 1}\left|\int_{T}^{T+c} \log \right| \zeta(1 / 2+i t)|d t|=h(T) \log T .
$$

Given arbitrary $A>0$, set

$$
h_{1}(T)=\sup _{t \geq T} h(t), \quad T>1,
$$




$$
\Delta=\Delta(T)=\frac{h_{1}(T)}{A} .
$$

Then $\Delta(T)$ decreases, and $\lim _{T \rightarrow \infty} \Delta(T)=0$. Hence there exists $T_{0}>1$ such that $\Delta(T) \leq 1$ for $T>T_{0}$. Then by (3.3)-(3.5), for $T>T_{0}$ we have

$$
\left|\int_{T}^{T+\Delta(T)} \log \right| \zeta(1 / 2+i t)|d t| \leq \Delta(T) A \log T .
$$

Thus

$$
\frac{1}{\Delta(T)} \int_{T}^{T+\Delta(T)} \log |\zeta(1 / 2+i t)| d t \geq-A \log T .
$$

That is, the mean value of $\log |\zeta(1 / 2+i t)|$ on $[T, T+\Delta(T)]$ is not less than $-A \log T$, so there exists $t_{0} \in[T, T+\Delta(T)]$ such that

$$
\log \left|\zeta\left(1 / 2+i t_{0}\right)\right| \geq-A \log T \text {. }
$$

Hence $\left|\zeta\left(1 / 2+i t_{0}\right)\right| \geq T^{-A}$. Then $F(T ; \Delta) \geq T^{-A}$. The proof is complete.

Acknowledgements. The author thanks the referee for valuable comments, Professor Ding Xiaqi for fruitful discussions, and Professor He Yuzan for translating the ref. [1].

\section{References}

[1] H. Cramér, Über die Nullstellen der Zetafunktion, Math. Z. 2 (1918), 237-241.

[2] M. Z. Garaev, Concerning the Karatsuba Conjectures, Taiwanese J. Math. 6 (2002), 573-580.

[3] A. A. Karatsuba, On lower estimates of the Riemann zeta function, Dokl. Akad. Nauk 376 (2001), 15-16.

[4] - Lower bounds for the maximum modulus of $\zeta(s)$ in small domains of the critical strip, Math. Notes 70 (2001), 724-726.

[5] J. E. Littlewood, On the zeros of the Riemann zeta function, Proc. Cambridge Philos. Soc. 22 (1924), 295-318.

6] E. C. Titchmarsh, The Theory of the Riemann Zeta Function, 2nd ed., revised by D. R. Heath-Brown, Clarendon Press, Oxford, 1986.

Academy of Mathematics and Systems Science

Chinese Academy of Sciences

Beijing 100080, P.R. China

E-mail: jxfsj@hotmail.com 